\title{
Three-Dimensional Waveguide Splitters Inscribed in Nd:YAG by Femtosecond Laser Writing: Realization and Laser Emission
}

\author{
Yuechen Jia, Chen Cheng, Javier Rodríguez Vázquez de Aldana, and Feng Chen, Senior Member, OSA
}

\begin{abstract}
In this paper, we report on a three-dimensional beam splitter monolithically integrated in an Nd:YAG laser crystal wafer by applying the direct femtosecond laser writing technique with the photonic-lattice-like cladding approach, capable of 1 to 2 and 1 to 4 beam splitting. Using the pump at $808 \mathrm{~nm}, 1064-\mathrm{nm}$ splitting lasers with slope efficiencies of $34 \%$ and $22 \%$ for 1 to 2 and 1 to 4 configurations were realized. This study paves a way to fabricate direct-pump compact laser devices in a single chip for light guiding and beam dividing in Nd:YAG crystals for various photonic applications.
\end{abstract}

Index Terms-Femtosecond laser writing, integrated optics, waveguide lasers, 3-D waveguide splitters.

\section{INTRODUCTION}

A $\mathrm{S}$ one of the essential and significant power division devices in integrated optical circuits and miniature photonic devices, the beam splitter based on the optical waveguide platform has attracted numerous attentions on account of its low propagation loss and discontinuity [1], [2]. Benefiting from this, it has been extensively applied in optical telecommunications, quantum computing, biophotonic sensing, information processing etc. [3]-[6]. Waveguide lasers, as ideal candidates of miniature light sources in compact and integrated circuits, have been developed in many active waveguide systems, ranging from visible to mid-infrared wavelength bands [7]-[18]. A few techniques have been developed to fabricate waveguides in dielectrics. Since 1996, the femtosecond laser writing has been widely applied to produce waveguides and other photonic devices in various transparent materials [19], [20]. One of the challenges of waveguide laser devices is the realization of flexible manipulation of the generated light-beam profiles for actual control of spatial properties of laser radiations. As a 3-D micromachining technique, the femtosecond laser writing enables on-demand construction of waveguides at arbitrary depths in-

Manuscript received July 9, 2015; revised September 18, 2015, October 30, 2015, and November 16, 2015; accepted November 21, 2015. Date of publication November 23, 2015; date of current version February 10, 2016. This work was supported by National Natural Science Foundation of China (No. 11274203) and Ministerio de Economía y Competitividad under Project FIS2013-44174-P, Spain.

Y. Jia, C. Cheng, and F. Chen are with the School of Physics, Shandong University, Jinan 250100, China, and also with the State Key Laboratory of Crystal Materials, Shandong University, Jinan 250100, China (e-mail: jiayuechen0521@163.com; chengch_sdu@163.com; drfchen@sdu.edu.cn).

J. R. Vázquez de Aldana is with the Laser Microprocessing Group, Departamento de Física Aplicada, Universidad de Salamanca, Salamanca 37008, Spain (e-mail: jrval@usal.es).

Color versions of one or more of the figures in this paper are available online at http://ieeexplore.ieee.org.

Digital Object Identifier 10.1109/JLT.2015.2503349 side the substrates. This advantage allows the development of innovative and unique device architectures, such as 3-D photonic lantern and 3-D waveguide splitters [20]-[25]. Nevertheless, the femtosecond-laser fabricated 3-D waveguide splitter structures were mostly realized in amorphous materials, such as glass. This is because the femtosecond laser pulses usually induce positive refractive index changes $(\Delta n>0)$ in glass, which results in guiding cores that are generally located just in the femtosecondlaser irradiated regions. In such cases, the geometries of the waveguide structures could be easily controlled. Conversely, in dielectric crystals, negative index changes $(\Delta n<0)$ are typically induced through the femtosecond laser modifications, therefore the waveguides are usually situated in the surrounding regions of the femtosecond-laser induced tracks. In addition, the refractive index changes induced by the femtosecond lasers are usually anisotropic, resulting in light guidance with polarization-sensitive features [22]-[26]. These behaviors bring out certain intractability for the 3-D guiding implementation in dielectric crystals [26]. Recently, by employing femtosecond laser micromachining, 2-D waveguide beam splitter lasers (within a plane) based on hexagonal photonic-lattice-like structures [27], surface cladding structures [28], and double-line configurations [29] have been realized in YAG crystals. In this study, we experimentally achieve the implementation of monolithic 3D waveguide beam splitters based on the photonic-lattice-like structures, which contains linear inscribed tracks packed in a hexagonal lattices with cores of unmodified Nd:YAG, capable of efficient light guiding. The on-demand introduction of axial track defects in the structures enables the light beam manipulation for 3-D beam splitting. Such a designable feature for beam manipulation in 3-D manner, in principle, paves a way to construct compact arbitrary devices for any purpose. Using direct-pump of the monolithic structures, the continuous wave (CW) 3-D waveguide splitting lasing (i.e., 1 to 2 or 1 to 4 ) at near infrared wavelength of $1064 \mathrm{~nm}$ has been obtained.

\section{EXPERIMENTS IN DETAILS}

The structures are fabricated in Nd:YAG laser crystal, one of the most favorable gain media for solid state lasers owing to its excellent fluorescence, physical and thermal properties. The Nd:YAG (doped by 1 at. $\% \mathrm{Nd}^{3+}$ ions) crystal wafer is cut with dimension of $10 \times 10 \times 2 \mathrm{~mm}^{3}$ and optically polished, the waveguide microstructures are inscribed along the length of $10 \mathrm{~mm}$. An amplified Ti:Sapphire femtosecond laser (Spitfire, Spectra Physics) that delivered linearly polarized pulses with a 


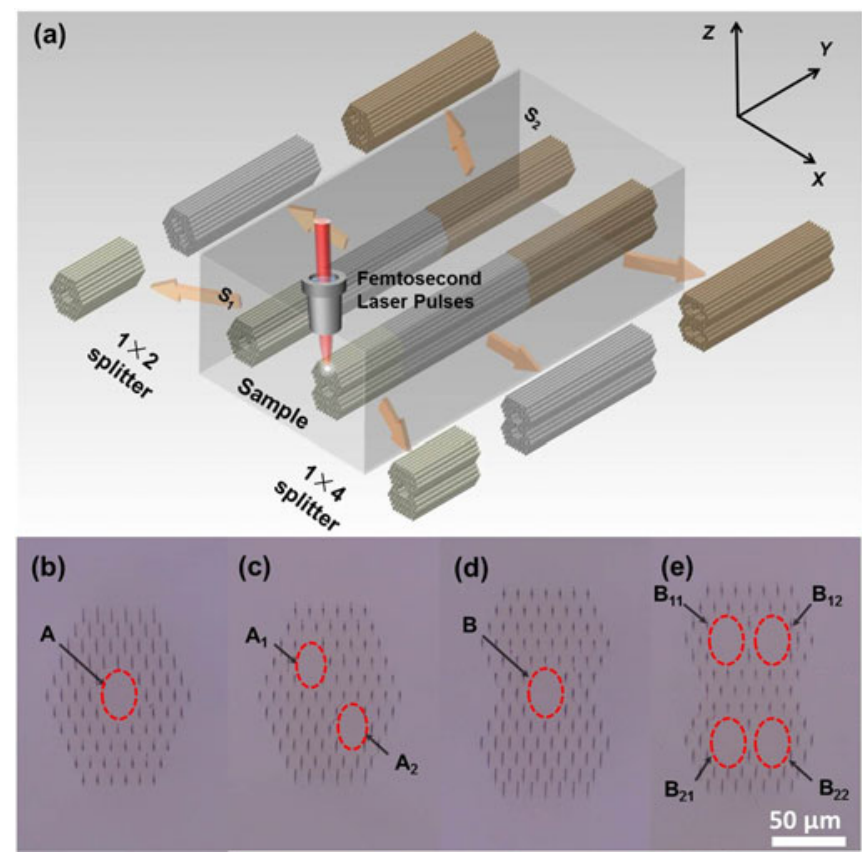

Fig. 1. (Color Online) (a) Schematic diagram of the 3-D photonic-latticelike microstructures. $S_{1}$ and $S_{2}$ represent the input and output facets, respectively. (b)-(e) Optical microscope images of the cross sections of the waveguide structures.

temporal duration of $120 \mathrm{fs}$, a central wavelength of $795 \mathrm{~nm}$ and operating at a repetition rate of $1 \mathrm{kHz}$, is used as the laser source. The beam is focused by a $40 \times$ microscope objective and the average power is reduced to $1.2 \mathrm{~mW}$ (measured after the focusing objective) by using a set of half-wave plate and linearpolarizing cube, and a calibrated neutral density filter. The laser irradiation is controlled with a mechanical shutter. The sample is placed on a XYZ micro-positioning stage that allows scanning the sample at constant velocity $(0.5 \mathrm{~mm} / \mathrm{s})$ while irradiating from the surface of $10 \times 10 \mathrm{~mm}^{2}$ with the femtosecond pulses at certain depth beneath the surface $(\sim 150 \mu \mathrm{m})$ : under our experimental conditions, a damage track is then produced with a transverse length of $10 \mu \mathrm{m}$. A number of parallel scans of the sample are performed, with a lateral separation of $10 \mu \mathrm{m}$, at different depths in order to obtain the desired photonic-lattice-like structures. The central core is approximately an elliptical region with area of $25(x) \times 30(z) \mu \mathrm{m}^{2}$. Fig. 1(a) shows the schematic diagram of 3-D photonic-lattice-like microstructures (i.e., 1 to 2 (output ports not within same plane) and 1 to 4 waveguide splitters). As we can see, two 3-D photonic-lattice-like microstructures (with guiding cores surrounded by hexagonally and double hexagonally arrayed track lattices) are fabricated in the sample by introducing specially designed defect tracks in the core regions at different parts of the whole prototypes. By combining three designed elements with smoothly changing guiding cores, which are connected in sequence, the 3-D 1 to 2 and 1 to 4 waveguide splitters are produced in the Nd:YAG crystal sample (the lengths of the three elements are 2, 4 and $4 \mathrm{~mm}$, respectively, for both 1 to 2 and 1 to 4 waveguide splitters). It is easy to find that arbitrarily complex structures could be designed by intro- ducing more intermediate elements with on-demand axial track defects at certain positions, which is an intriguing and promising technique for various photonic applications. Fig. 1(b)-(e) depict the optical microscope images of the waveguide end facets, in which $\mathrm{A}, \mathrm{B}$ (input) and $\mathrm{A}_{1}-\mathrm{A}_{2}, \mathrm{~B}_{12}-\mathrm{B}_{22}$ (output) indicate the different ports of the structures $\left(B_{1}\right.$ and $B_{2}$ are for the second part of the 1 to 4 waveguide splitter, which is not shown in Fig. 1). The transverse deviation displacements of the output channels in the two different configurations are calculated to be 26 (the distances between centers of $A_{1}$ and $A$ ) and $36 \mu \mathrm{m}$ (the distances between centers of $\mathrm{B}_{21}$ and $\mathrm{B}$ ). For comparison, another 10-mm long photonic-lattice-like structure with homogeneous profile (same as the 2-mm element of 1 to 2 waveguide splitter) is manufactured in this sample as well. Such a straight guiding structure is used as a reference.

The laser operation experiments are performed by utilizing an end pumping system at room temperature. A polarized light beam at a wavelength of $808 \mathrm{~nm}$ is generated from a tunable CW Ti:Sapphire laser (Coherent MBR PE). A convex lens with focus length of $25 \mathrm{~mm}$ is used to couple the laser beam into the photonic structures. An input mirror (with a transmission of $98 \%$ at $808 \mathrm{~nm}$ and a reflectivity of $\sim 99 \%$ at $1064 \mathrm{~nm}$ ) and an output mirror (with a reflectivity of $~ 99 \%$ at $808 \mathrm{~nm}$ and $60 \%$ at $1064 \mathrm{~nm}$, respectively) are adhered to the input and output end facets of the waveguides respectively, constructing the FabryPerot lasing resonant cavity. Here, a set of mechanical bindings are employed to make the mirrors closely attached to the facets of the waveguide sample. The generated lasers are collected by utilizing a $20 \times$ microscope objective lens (N.A. $=0.4$ ) and imaged by using an infrared charge-coupled device. A dichroic beam splitter is used to separate the residual non-absorbed 808 -nm pump radiation. We use a spectrometer with resolution of $0.2 \mathrm{~nm}$ to analyze the emission spectra of the laser beams.

\section{RESUlTS AND DISCUSSION}

The refractive index change in the femtosecond laser inscribed tracks (the contrast between individual modification and the unprocessed crystal) is determined to be $\Delta n \approx-4 \times 10^{-3}$. Briefly, the refractive index map is constituted by the superposition of the damage induced refractive index modification (reduction) and the stress field induced refractive index map (refractive index increment at compressed volumes). The first is obtained from the fluorescence images based on the fluorescence intensity as the fluorescence intensity reduction in respect to the bulk material is, in a first order approximation, assumed proportional to the laser-induced damage in the Nd:YAG network. The conversion of laser induced damage into refractive index reduction is performed by a reference Nd:YAG sample that contains different damage tracks fabricated under the same conditions of femtosecond laser irradiation. The stress-induced refractive index map has been calculated from the fluorescence map obtained in terms of the induced spectral shift of $\mathrm{Nd}^{3+}$ fluorescence lines. From the spectral shift, one calculate the spatial variation of residual stress and from this the relative change in the unit cell volume (i.e., the lattice dilatation/densification). 


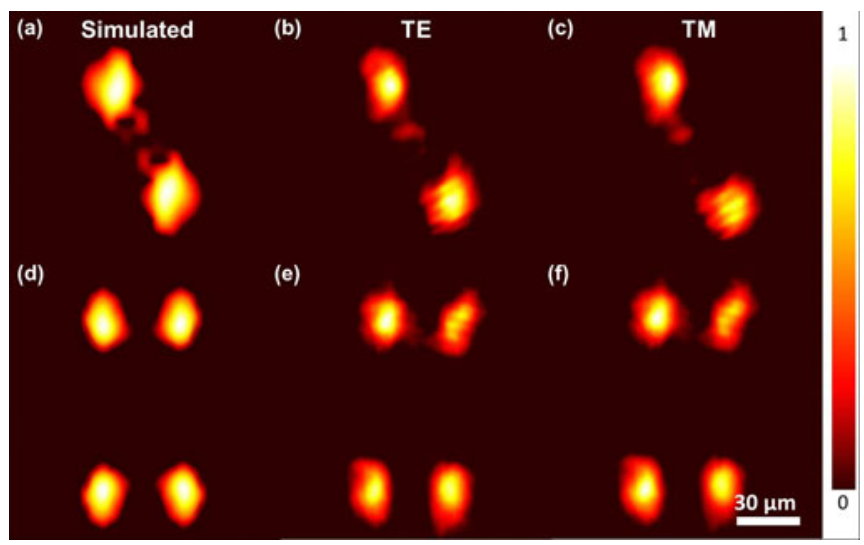

Fig. 2. (Color Online) Simulated beam modal profiles of 1064-nm light propagating through (a) 1 to 2 and (d) 1 to 4 waveguide splitter and the measured (b,e) TE and (c,f) TM modes at lasing wavelength of $\sim 1064 \mathrm{~nm}$, respectively. The input laser is with a Gaussian beam profile.

Finally, the refractive index modification is calculated by applying the Claussius-Mossitti approximation [27]. With this value the beam modal profiles of 1064-nm light propagating through these 3-D photonic-lattice-like microstructures are calculated by Rsoft software based on the finite-difference beam propagation method, shown in Fig. 2(a) (from $A$ to $A_{1}-A_{2}$ ) and 2(d) (from $\mathrm{B}$ to $\left.\mathrm{B}_{11}-\mathrm{B}_{22}\right)$. Fig. 2(b) and (c) ((e) and (f)) depict the 1 to 2 (1 to 4 ) waveguide splitter laser profiles of the measured TE and TM modes at lasing wavelength of $\sim 1064 \mathrm{~nm}$, respectively, which are in good agreement with the simulated mode data. It should be noted that the measured intensity splitting ratios are 0.51:0.49 for the two arms of the 1 to 2 splitter, and 0.255:0.255:0.255:0.235 for the four arms of 1 to 4 splitter, exhibiting excellent 3-D beam splitting performances with a great equalization of the output beams. The non-perfect splitting ratios are mainly due to the deviations induced by femtosecond laser writing during the fabrication process. In addition, the total attenuations of the 3-D waveguide splitters (including splitting losses and guiding propagation losses) at $1064 \mathrm{~nm}$ are determined to be 0.5 and $0.8 \mathrm{~dB}$ for 1 to 2 and 1 to 4 waveguide splitters, respectively. The measured value for the homogeneous photonic-lattice-like waveguide is also approximately $\sim 0.5 \mathrm{~dB}$, showing that, in 1 to 2 splitter regime, the axial defects do not introduce additional losses, and the beam manipulation can be implemented in the photonic-lattice-like structures efficiently.

Fig. 3 shows the output laser powers at $1064 \mathrm{~nm}$ as a function of incident pump power for the 3-D 1 to 2 and 1 to 4 waveguide splitters, respectively. For comparison, the lasing performance curve for the reference structure is also depicted in this figure. As the incident pump power is above a threshold $(\sim 90 \mathrm{~mW})$, lasing oscillations are observed in all the photonic-lattice-like structure systems. For the 1 to 2 waveguide splitter structure, the laser shows a slope efficiency of $34 \%$ and a maximum output power of $333 \mathrm{~mW}$. For the 1 to 4 waveguide splitter, the laser operates with a slope efficiency of $22 \%$, climbing to a maximum output power of $217 \mathrm{~mW}$ in case of an incident pump power of $1.06 \mathrm{~W}$. Compared with the lasing performance based on the reference structure, i.e., a straight photonic-lattice-like structure, (with slope efficiency of $52 \%$ and maximum output power of

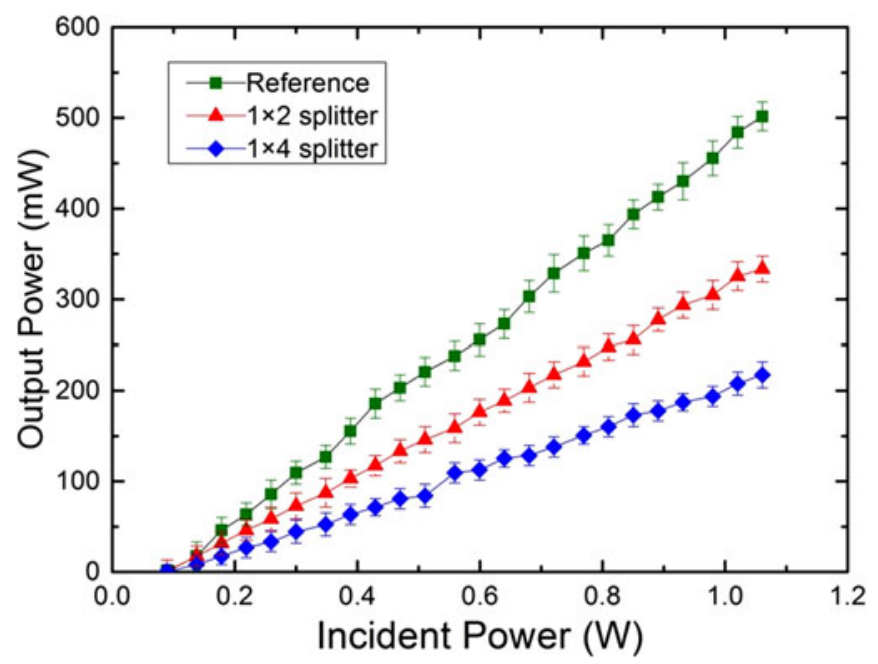

Fig. 3. (Color Online) Output laser powers at $1064 \mathrm{~nm}$ as a function of incident pump power for the waveguide splitters and the reference structure.

$502 \mathrm{~mW}$ ), there is a $33 \%$ and $56 \%$ decrease of the maximum output power for the 3-D 1 to 2 and 1 to 4 beam splitters, respectively. This should be mainly attributed to the mismatching of the pump and laser modes during the waveguide laser excitation and the additional bending losses from the split lasing cavity, i.e., non-perfect Fabry-Perot cavity, since the attenuation from the introduction of axial defects are negligible in passive regime. The bending losses (relevant to the size, shape and geometry of the laser resonate cavity) here, partly induced by the unsmooth bends at the joint positions, have a huge impact on the laser performance (the larger deviation displacements lead to greater additional attenuations), which could be improved by smoothing the curved part of the waveguide. It is worth mentioning that the coupling losses are not excluded from the calculation of the incident power in Fig. 3. Hypothetically, the slope efficiency of the straight reference structure could be increased to as high as $65 \%$ if the coupling efficiency of the pump beam and the waveguide mode profile is considered (estimated to be $\sim 80 \%$ ).

In addition, the thorough information of the pumping polarization effects of the waveguide splitter laser powers has been investigated by measuring the all-angle waveguide laser output power along the transverse plane under maximum $808 \mathrm{~nm}$ pumping power. It is found that the waveguide laser output power was independent of the polarization of the pump light (i.e., for any transverse polarization of the input light, the waveguide laser output power is almost same), indicating a perfectly isotropic light confinement performance. This feature is advantageous for unpolarized optical pump by diodes for high-power waveguide laser generation. In addition, our investigations have also indicated that, for the optical pumping with linearly polarized light at $808 \mathrm{~nm}$, the lasers at $1064 \mathrm{~nm}$ are with the same polarization to the pump beams. This means that the 1064$\mathrm{nm}$ output waveguide laser through the guiding structure well preserves the original polarization of the $808-\mathrm{nm}$ pump beam, which is in agreement with the previous experimental results in Nd:YAG bulk materials [30]. In fact, the polarization of the generated waveguide lasers also depends on the waveguide 
supporting configurations. For example, the laser-written dualline waveguides in Nd:YAG only support TM polarized modes, in which the waveguide lasers are always with vertical polarization at optical pump [9].

\section{CONCLUSION}

In conclusion, we have designed and fabricated 3-D waveguide splitter structures in a Nd:YAG laser crystal wafer by employing the direct femtosecond laser writing. The integration of a few specially designed photonic-lattice-like elements with defect lines has been demonstrated to be an adaptable and simple way to build compact 3-D photonic-lattice-like structures capable of producing efficient beam divisions. In addition, the splitting lasers at $1064 \mathrm{~nm}$ has been achieved demonstrating that, based on the proposed monolithic photonic-lattice-like structures arbitrarily complex 3-D beam manipulation devices could be implemented. This study paves a way to produce direct-pump compact integrated laser devices on chip scales for light guiding, laser action and beam splitting in dielectric laser crystals for various photonic applications.

\section{REFERENCES}

[1] K. Tanaka and M. Tanaka, "Simulations of nanometric optical circuits based on surface plasmon polariton gap waveguide," Appl. Phys. Lett., vol. 82, no. 8, pp. 1158-1160, Feb. 2003.

[2] C. Manolatou, S. G. Johnson, S. Fan, P. R. Villeneuve, H. A. Haus, and J. D. Joannopoulos, "High-density integrated optics," J. Lightw. Technol., vol. 17, no. 9, pp. 1682-1692, Sep. 1999.

[3] J. Porquea, P. Coudraya, R. Chartersb, K. Kribicha, P. Etiennec, and Y. Moreaua, "WDM based on multimode interference-coupler built in an organic-inorganic material," Opt. Commun., vol. 183, nos. 1-4, pp. $45-49$, Sep. 2000

[4] L. Sansoni, F. Sciarrino, G. Vallone, P. Mataloni, A. Crespi, R. Ramponi, and R. Osellame, "Polarization entangled state measurement on a chip," Phys. Rev. Lett., vol. 105, no. 20, art. no. 200503, Nov. 2010.

[5] A. Brandenburg, R. Krauter, C. Künzel, M. Stefan, and H. Schulte, "Interferometric sensor for detection of surface-bound bioreactions," Appl. Opt., vol. 39, no. 34, pp. 6396-6405, Dec. 2000.

[6] C. Zou, F. Sun, C. Dong, X. Ren, J. Cui, X. Chen, Z. Han, and G. Guo, "Broadband integrated polarization beam splitter with surface plasmon," Opt. Lett., vol. 36, no. 18, pp. 3630-3632, Sep. 2011.

[7] J. I. Mackenzie, "Dielectric solid-state planar waveguide lasers: A review," IEEE J. Sel. Topic Quantum Electron., vol. 13, no. 3, pp. 626-637, May 2007.

[8] C. Grivas, "Optically pumped planar waveguide lasers, Part I: Fundamentals and fabrication techniques," Prog. Quantum Electron., vol. 35, no. 6, pp. 159-239, Nov. 2011.

[9] T. Calmano and S. Mueller, "Crystalline waveguide lasers in the visible and near-infrared spectral range," IEEE J. Sel. Topic Quantum Electron., vol. 21, no. 1, art. no. 1602213, Jan./Feb. 2015.

[10] S. Müller, T. Calmano, P. Metz, N. O. Hansen, C. Kränkel, and G. Huber, "Femtosecond-laser-written diode-pumped Pr:LiYF 4 waveguide laser," Opt. Lett., vol. 37, no. 24, pp. 5223-5225, Dec. 2012.

[11] C. Grivas, C. Corbari, G. Brambilla, and P. G. Lagoudakis, "Tunable, continuous-wave Ti:sapphire channel waveguide lasers written by femtosecond and picosecond laser pulses," Opt. Lett., vol. 37, no. 22, pp. 4630-4632, Nov. 2012.

[12] T. Calmano, J. Siebenmorgen, A. G. Paschke, C. Fiebig, K. Paschke, G. Erbert, K. Petermann, and G. Huber, "Diode pumped high power operation of a femtosecond laser inscribed Yb:YAG waveguide laser," Opt. Mater. Exp., vol. 1, no. 3, pp. 428-433, Jul. 2011.

[13] A. Okhrimchuk, V. Mezentsev, A. Shestakov, and I. Bennion, "Low loss depressed cladding waveguide inscribed in YAG:Nd single crystal by femtosecond laser pulses," Opt. Exp., vol. 20, no. 4, pp. 3832-3843, Feb. 2012.
[14] G. Salamu, F. Jipa, M. Zamfirescu, and N. Pavel, "Laser emission from diode-pumped Nd:YAG ceramic waveguide lasers realized by direct femtosecond-laser writing technique," Opt. Exp., vol. 22, no. 5, pp. 5177-5182, Mar. 2014.

[15] N. Pavel, G. Salamu, F. Jipa, and M. Zamfirescu, "Diode-laser pumping into the emitting level for efficient lasing of depressed cladding waveguides realized in $\mathrm{Nd}: \mathrm{YVO}_{4}$ by the direct femtosecond-laser writing technique," Opt. Exp., vol. 22, no. 19, pp. 23057-23065, Sep. 2014.

[16] Y. Ren, G. Brown, A. Ródenas, S. Beecher, F. Chen, and A. K. Kar, "Midinfrared waveguide lasers in rare-earth-doped YAG," Opt. Lett., vol. 37, no. 16, pp. 3339-3341, Aug. 2012.

[17] D. G. Lancaster, S. Gross, H. Ebendorff-Heidepriem, A. Fuerbach, M. H. Withford, and T. M. Monro, "Crystalline $\mathrm{Pr}_{\mathrm{SrAl}} \mathrm{Sr}_{12} \mathrm{O}_{19}$ waveguide laser in the visible spectral region," Opt. Lett., vol. 36, no. 23, pp. 4620-4622, Dec. 2011.

[18] J. R. Macdonald, S. J. Beecher, P. A. Berry, K. L. Schepler, and A. K. Kar, "Compact mid-infrared Cr:ZnSe channel waveguide laser," Appl. Phys. Lett., vol. 102, no. 16, art. no. 161110, Apr. 2013.

[19] K. M. Davis, K. Miura, N. Sugimoto, and K. Hirao, "Writing waveguides in glass with a femtosecond laser," Opt. Lett., vol. 21, no. 21, pp. 1729-1731, Nov. 1996.

[20] R. R. Thomson, T. A. Birks, S. G. Leon-Saval, A. K. Kar, and J. BlandHawthorn, "Ultrafast laser inscription of an integrated photonic lantern," Opt. Exp., vol. 19, no. 6, pp. 5698-5705, Mar. 2011.

[21] T. A. Birks, I. Gris-Sánchez, S. Yerolatsitis, S. G. Leon-Saval, and R. R. Thomson, "The photonic lantern," Adv. Opt. Photon., vol. 7, no. 2, pp. 107-167, Jun. 2015.

[22] R. Osellame, G. Cerullo, and R. Ramponi, Femtosecond-Laser Micromachining: Photonic Microfluidic Devices Transparent Materials. Berlin, Germany: Springer, 2012.

[23] S. Nolte, M. Will, J. Burghoff, and A. Tuennermann, "Femtosecond waveguide writing: a new avenue to three-dimensional integrated optics," Appl. Phys. A, vol. 77, no. 1, pp. 109-111, Jun. 2003.

[24] K. Sugioka, J. Xu, D. Wu, Y. Hanada, Z. Wang, Y. Cheng, an K. Midorikawa, "Femtosecond laser 3D micromachining: A powerful tool for the fabrication of microfluidic, optofluidic, and electrofluidic devices based on glass," Lab Chip., vol. 14, no. 18, pp. 3447-3458, 2014.

[25] R. R. Gattass and E. Mazur, "Femtosecond laser micromachining in transparent materials," Nat. Photon., vol. 2, no. 4, pp. 219-225, Apr. 2008.

[26] F. Chen and J. R. Vázquez de Aldana, "Optical waveguides in crystalline dielectric materials produced by femtosecond-laser micromachining," Laser Photon. Rev., vol. 8, no. 2, pp. 251-275, Mar. 2014.

[27] Y. Jia, C. Cheng, J. R. Vázquez de Aldana, G. R. Castillo, B. del Rosal Rabes, Y. Tan, D. Jaque, and F. Chen, "Monolithic crystalline cladding microstructures for efficient light guiding and beam manipulation in passive and active regimes," Sci. Rep., vol. 4, art. no. 5988, Aug. 2014.

[28] H. Liu, C. Cheng, C. Romero, J. R. Vázquez de Aldana, and F. Chen, "Graphene-based Y-branch laser in femtosecond laser written Nd:YAG waveguides," Opt. Exp., vol. 23, no. 8, pp. 9730-9735, Apr. 2015.

[29] T. Calmano, C. Kränkel, and G. Huber, "Laser oscillation in Yb:YAG waveguide beam-splitters with variable splitting ratio," Opt. Lett., vol. 40 no. 8, pp. 1753-1756, Apr. 2015.

[30] N. V. Kravtsov, E. G. Lariontsev, and N. I. Naumkin, "Dependence of polarisation of radiation of a linear Nd:YAG laser on the pump radiation polarization," Quant. Electron., vol. 34, no. 9, pp. 839-842, May 2004.

Yuechen Jia received the B.S. and Ph.D. degrees from Shandong University, Jinan, China, in 2010 and 2015, respectively. He is currently working as a Postdoctoral Fellow at the University of Freiburg, Breisgau, Germany. He is an Alexander von Humboldt Research Fellow.

His current research interests include fabrication of optical waveguides and micro cavities in laser and nonlinear materials.

Chen Cheng received the B.S. degree from Shandong Normal University, Jinan, China, in 2013. He is currently working toward the Ph.D. degree at Shandong University, Jinan, China.

His current research interests include fabrication of optical waveguides in optical crystals by using femtosecond laser writing. 
Javier Rodríguez Vázquez de Aldana received the B.S. and Ph.D. degrees from the University of Salamanca, Spain, in 1997 and 2001, respectively. $\mathrm{He}$ is currently an Associate Professor of the Science Faculty, University of Salamanca, Salamanca, Spain.

His research interests include the interaction of intense femtosecond pulses with materials and its application to the fabrication of photonic devices. He is a Member of the Laser Microprocessing Research Group, and is also technical and scientific advisor of the Laser Facility at the University of Salamanca.
Feng Chen received the B.S. and Ph.D. degrees from Shandong Normal University and Shandong University, Jinan, China, in 1997 and 2002, respectively. In 2002, he was a Lecturer of physics at Shandong University, when he finished his doctoral dissertation on the ion-implanted optical waveguides and their applications in opto-electronics. From 2003 to 2005, he was engaged in research on discrete solitons in nonlinear periodic waveguide arrays or lattices as an Alexander von Humboldt Research Fellow in the Clausthal University of Technology, Germany. In 2004, he became an Associate Professor at Shandong University. In 2006, he became a Professor of physics at Shandong University, where he is currently with the School of Physics and State Key Laboratory of Crystal Materials.

His current research interests include optical waveguides devices produced by energetic ion beam irradiation and ultrafast laser writing, waveguide lasers, and nonlinear optics. Prof. Chen is currently a Senior Member of the Optical Society of America, a Fellow of the Institute of Physics, U.K., and a Director of Chinese Physical Society. He is also an Associate Editor of Optical Engineering and an Editorial Board Member of Scientific Reports. 